Pathologe 2008 · [Suppl 2] 29:187-190

DOI 10.1007/s00292-008-1030-2

Online publiziert: 6. September

(c) Springer Medizin Verlag 2008

\author{
H. Lage ${ }^{1} \cdot$ P. Surowiak ${ }^{2} \cdot$ P.S. Holm ${ }^{3}$ \\ ${ }^{1}$ Institut für Pathologie, Charité-Universitätsmedizin Berlin, Campus Mitte \\ ${ }^{2}$ Institut für Histologie und Embryologie, Universität Breslau, Polen \\ ${ }^{3}$ Institut für Experimentellelle Onkologie und Therapieforschung, TU München
}

\title{
YB-1 als potenzielles Ziel für die Tumortherapie
}

Bei YB-1 (Y-Box Protein 1) handelt es sich um ein $42 \mathrm{kDa}$ großes Protein, welches sich aus 322 Aminosäureresten zusammensetzt. Die Namensgebung von YB-1, dessen $19 \mathrm{~kb}$ großes, 8 Exons aufweisendes Gen auf der chromosomalen Region 1p34 lokalisiert ist, beruht auf seiner Identifizierung als Protein, das an die Y-Box in der Promotorregion humaner MHC-Klasse-II-KomplexGene bindet [5]. Bei einer Y-Box handelt es sich um eine invertierte Version der klassischen CCAAT-Box, die aus den Promotorregionen vieler eukaryontischer Gene bekannt ist.

Das zur Familie der Cold-Shock-Domain- (CSD-)Proteine gehörende YB-1 hat pleiotrope biologische Funktionen bei der Regulation von Transkription, Translation, DNA-Reparatur und bei zellulären Reaktionen auf diverse Stressfaktoren [18, 30]. Es setzt sich aus drei unterschiedlichen Domänen zusammen: $a$ einer bei CSDProteinen variablen $\mathrm{N}$-terminalen Domäne (A/P-Domäne), $b$ der hochkonservierten CSD sowie $c$ einer C-terminalen Domäne (CTD). Funktionell ist die A/P-Domäne in die Regulation von Transkriptionsvorgängen involviert [18]. Die CSD kann an Nukleinsäuren, wie DNA und RNA Oligonukleotiden, dsDNA und ssDNA binden. Sie weist zudem zwei konservierte Ribonucleoprotein-Particles- (RNP-)Motive auf, die Funktionen beim intrazellulären Transport sowie bei der Kontrolle von Translationsvorgängen wahrnehmen. Die CTD besteht aus einem Bereich aus alternierenden sauren und basischen Aminosäuren („,B/A repeats"), der sich für Protein-Protein-Interaktionen verantwortlich zeigt. „B/A repeats" stellen ein gemeinsames Merkmal vieler Proteine dar, die über Bindung an Ribonukleoproteinkomplexe zwischen dem zytoplasmatischen Zellkompartement und dem Zellkern translokalisiert werden [24]. Darüber hinaus konnten in der CTD zwei Regionen identifiziert werden, die die subzelluläre Lokalisation von YB-1 regulieren: $a$ die NLS („,noncanonical nuclear localization signal“) und $b$ die CRS („cytoplasmic retention site") [1]. Die subzelluläre Translokalisation von YB-1 in den Zellkern wird zudem über eine Interaktion der CSD mit der CTD unterstützt [13].

\section{Expression von YB-1 in neoplastischen Zellen}

YB-1 konnte in unterschiedlichen Tumoren nachgewiesen werden, z. B. in kolorektalen Karzinomen [27], Mammakarzinomen $[2,7,8,12]$, Ovarialkarzinomen [14, 23], Prostatakarzinomen [9], Bronchialkarzinomen [26], Osteosarkomen [21], Synovialsarkomen [22] sowie kindlichen Glioblastomen [6] und Ewing-Sarkomen [30]. Aufgrund des häufigen Vorhandenseins von YB-1 in Tumoren scheint YB-1 dort eine wichtige Rolle zu spielen. Interessanterweise konnte in einem YB1 überexprimierenden transgenen Mausmodell gezeigt werden, dass YB-1 in 100\% der untersuchten Tiere das Wachstum invasiver Mammakarzinome induzierte [3]. Die Bedeutung von YB-1 in Tumoren soll daher am Beispiel des Mammakarzinoms etwas detaillierter diskutiert werden.

In mehreren Studien konnte gezeigt werden, dass YB-1 im Mammakarzinom exprimiert wird. Dabei zeigte sich, dass eine starke gesamtzelluläre YB-1-Expression mit einer schlechteren Überlebenszeit korreliert [12] bzw. eine nukleäre YB-1Expression mit ungünstiger progressionsfreier und Gesamtüberlebenszeit assoziiert ist [7]. Beide Studien kommen zu dem Schluss, dass YB-1 einen unabhängigen prognostischen und prädiktiven Marker für das Mammakarzinom darstellt. Anzumerken ist, dass eine andere Studie - allerdings mit einer sehr geringen Fallzahl von nur 31 Proben - keine Korrelationen zwischen nukleärer YB-1-Expression und Überlebenszeit finden konnte [25]. Ei-

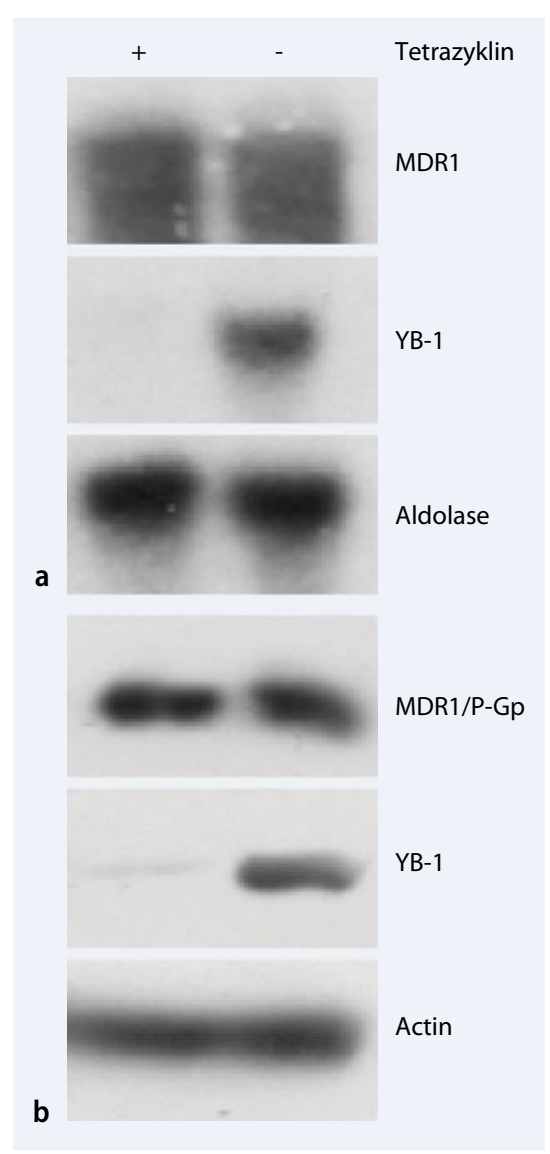

Abb. $1 \Delta$ RNAi-vermittelte Inhibition der YB-1Expression in chemoresistenten, MDR1-positiven Tumorzellen. Nach Gabe von Tetrazyklin synthetisieren die Tumorellen anti-YB-1-siRNAs, wodurch die YB-1-Expression inhibiert wird. Die Expressionstärke der MDR1-mRNA wird durch die YB-1-Inhhibition nicht beeinflusst. a Northern Blot. b Western Blot 


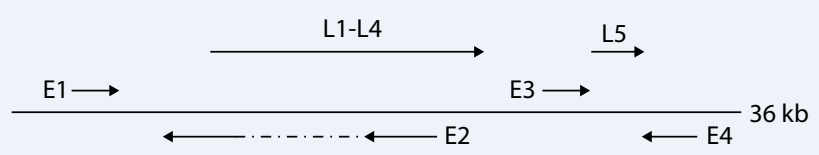

Abb. $2 \triangle$ Schematische Darstellung der genomischen Struktur eines Adenovirus Typ 5. E1-E4: Früh exprimierte Gene („early genes“); die E1-Region setzt sich dabei aus den Unterregionen E1A und E1B zusammen. L1-L5: spät exprimierte Gene („late genes"). Therapeutische Transgene, z. B. auch RNAivermittelnde Sequenzen ersetzen die Regionen E1 und E3. Im wildtyp Adenovirus Typ 5 ist die E1Region für die Replikation essenziell. YB-1 kann die Funktion des E1A-Proteins übernehmen und eine Virusreplikation bewirken

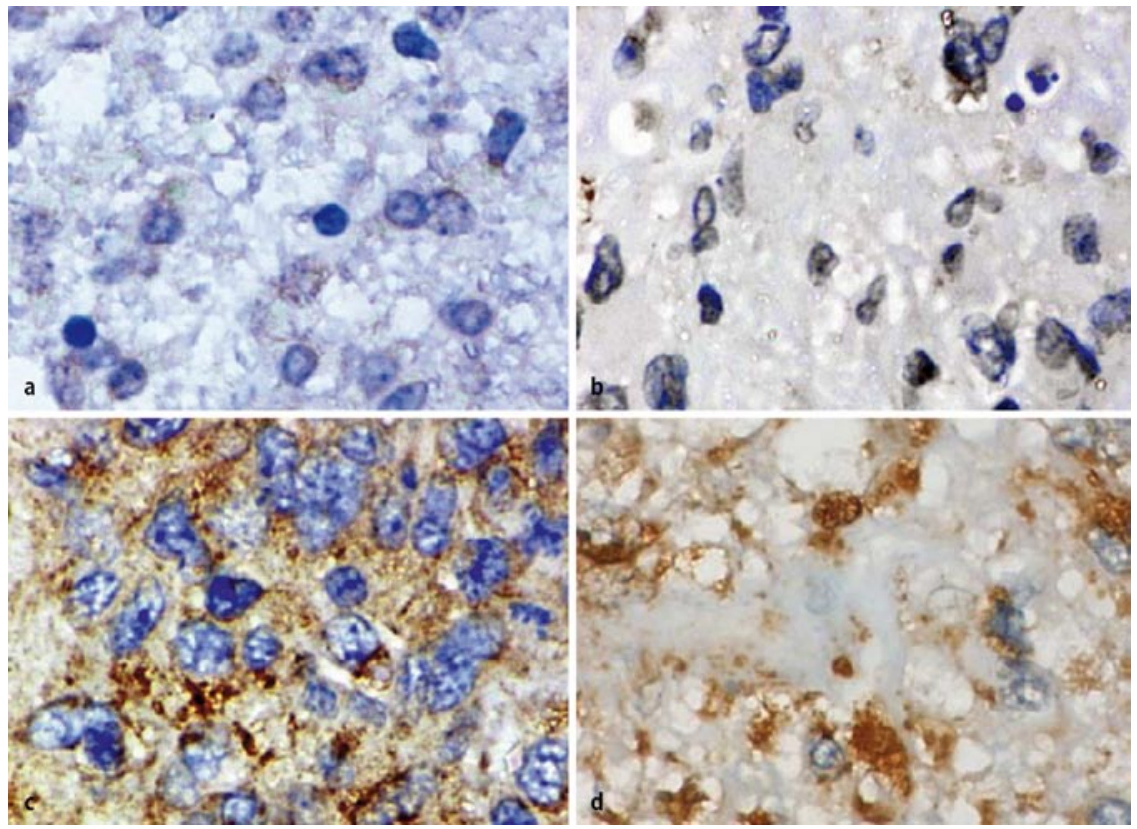

Abb. $3 \Delta$ YB-1-Expressionsmuster im Glioblastom und normalen Gehirn. a Normalgewebe ist komplett negativ für YB-1. b Glioblastom ohne YB-1-Expression. c Glioblastom mit zytoplasmatisch lokalisiertem YB-1. d Glioblastom mit zytoplasmatischer und nukleärer YB-1-Lokalisation

ne mögliche Erklärung für diese Korrelationen wurde in vitro dadurch erbracht, dass YB-1 eine Überexpression von EGFR und Her-2 in Mammakarzinomzellen induziert [29]. Diese Daten wurden in einer anderen Studie bestätigt, jedoch nur in Östrogenrezeptor- $\alpha$ - (ER $\alpha$-)postiven Mammakarzinomzellen, in denen YB-1 auch die Expression von ERa hoch reguliert [7]. In Patientenproben konnte dieser Zusammenhang für Her-2, allerdings nicht für EGFR, bestätigt werden [7]. Nukleäres YB-1 scheint daher als mögliches Target zur Inhibition Her-2 und ERa vermittelter Proliferations- bzw. Apoptosehemmungssignalen in Frage zu kommen; insbesondere bei Resistenz gegenüber gegen Her-2 gerichtete Therapeutika wie Trastuzumab (Herceptin).

Im Mammakarzinom wurde eine weitere interessante Beobachtung bezüglich
YB-1 gemacht. Nukleäres YB-1 - aber nicht zytoplasmatisch lokalisiertes - korreliert mit der Expression des den klasPhänotyp verursachenden Transmembrantransporters MDR1/P-Glykoprotein (MDR1/P-Gp) [2, 8, 25]. Da In-vitroUntersuchungen zudem gezeigt haben, dass YB-1 den Promotor des MDR1/PGp Gens aktivieren kann [28], erschien YB-1 auch als mögliches Target zur Überwindung von MDR geeignet zu sein. Es wurde bisher allerdings noch nie mittels YB-1-cDNA-Transfektion überzeugend gezeigt, dass YB-1 tatsächlich in der Zelle eine verstärkte $\mathrm{MDR} 1 / \mathrm{P}-\mathrm{Gp}$-Expression induziert und damit einen MDR-Phänotyp auf eine Tumorzelle übertragen kann. Zur Klärung dieser Frage wurden unterschiedliche In-vitro-Modelle etabliert [16]. In diesen YB-1-positiven MDR- und sischen Multidrug-Resistenz- (MDR-)
Nicht-MDR-Tumozellinien lässt sich die Expression von YB-1 mittels eines induzierbaren RNA-Interferenz- (RNAi-)Systems [19] komplett inhibieren, d. h. durch Zugabe von Tetrazyklin werden in den Tumorzellen RNAi-vermittelnde anti-YB-1siRNA- (small interferring RNA-)Moleküle gebildet. - Abb. 1 zeigt am Beispiel einer Tumorzellinie (EPG85-257RDB), dass es zwar möglich ist, die YB-1-Expression komplett zu inhibieren, dass diese Inhibition allerdings keinen Einfluss auf die MDR1/P-Gp-Expression oder den MDR-Phänotyp hat. Im Gegensatz dazu konnte mit einem onkolytischen YB-1-abhängigen Adenovirus eine Inhibition der MDR1/P-Gp-Expression beobachtet werden, wobei der biologische Mechanismus allerdings bisher nicht geklärt ist [20]. Eine Eignung von YB-1 als mögliches Target zur Überwindung von MDR erscheint vor diesen Hintergründen fraglich.

\section{Die Rolle von YB-1 bei Virotherapie und viraler Gentherapie}

Ein weiterer wichtiger Aspekt der YB-1Biologie besteht darin, dass YB-1 an der Replikation von Adenoviren, die in der Gentherapie genutzt werden, beteiligt ist. Für gentherapeutische Strategien werden in der Regel Adenoviren Typ 5 genutzt, die aufgrund sicherheitsrelevanter Gesichtspunkte Deletionen im genomischen Bereich $\mathrm{E}_{1}\left(\Delta \mathrm{E}_{1}\right)$ aufweisen. Nach Eindringen eines Adenovirus in eine Zelle aktivieren die Genprodukte der viralen E1-Region die Gene der viralen E2-Region, wodurch eine Replikation der Viren und damit ein Lyse der Wirtszellen initiiert wird (• Abb.2). Die $\Delta$ E1-Deletion verhindert somit eine unkontrollierte Replikation der Viren. Zellen, die bei einer systemischen Applikation nicht mittels der viralen Gentherapie erreicht werden sollen, sollen auf diese Art und Weise vor Beeinträchtigungen geschützt werden. Interessanterweise konnte gezeigt werden, dass $\Delta$ E1-Adenoviren in Zellen mit nukleären YB-1 replizieren können. Dabei kann YB-1 die Funktion eines Genprodukts der viralen E1-Region, exakt des E1A-Proteins, übernehmen und eine virale Replikation über die Aktivierung des späten E2APromotors (E2A late promoter) veranlas- 
sen $[10,11]$; YB-1 weist somit eine biologische Aktivität, die der des viralen E1AProteins entspricht, auf (E1A-like activity). Das heißt, YB-1 kann einen „onkolytischen“ Effekt in Tumorzellen, die nukleär positiv für YB-1 sind, bewirken.

Der YB-1-abhängige „onkolytische“ Effekt konnte kürzlich in einer In-vivo-Studie deutlich bestätigt werden [4]. Nukleär YB-1-positive Glioblastomxenografts wurden mittels der Standardtherapie des Glioblastoms einer Radiatio, einer Virustherapie oder einer kombinierten Radiatio/Virustherapie behandelt. Es zeigte sich dabei ein vergleichbarer therapeutischer Effekt der Radiatio und der Virustherapie. Die Kombinationstherapie zeigte jedoch einen drastisch verstärkten Behandlungserfolg, bei dem sich praktisch kein Tumorwachstum mehr nachweisen ließ. Nukleär lokalisiertes YB-1 ist somit die Voraussetzung für einen onkolytischen Viruseffekt in vivo und stellt damit das zelluläre Target des viralen Therapieansatzes dar. Obwohl in kindlichen Glioblastomen bereits nachgewiesen wurde, dass YB-1 dort exprimiert wird und nukleär lokalisiert einen ungünstigen Prognosefaktor darstellt, sind entsprechende Untersuchungen in Glioblastomen erwachsener Patienten bisher noch nicht abgeschlossen. Dass dies aber der Fall ist, haben eigene Untersuchungen bereits gezeigt (• Abb. 3). Aufbauend auf die bisherigen Daten befindet sich eine Phase-I-Studie zur YB-1-abhängigen Virotherapie des Glioblastoms in Vorbereitung, die aller Voraussicht nach im Jahre 2009 beginnen soll.

\section{Verstärkter YB-1-abhängiger RNAi-Effekt}

Darüber hinaus zeigen erste Ergebnisse von In-vitro-Untersuchungen, dass sich eine nukleäre Präsens von YB-1 für eine verstärkte Effizienz therapeutischer RNAiStrategien nutzen lässt [17]. Aufgrund des bisher nicht gelösten Problems der Aufnahme von therapeutischen siRNA-Molekülen in Tumorzellen, sind alternative Strategien entwickelt worden, die darauf basieren, DNA in Tumorzellen einzubringen, deren Sequenz die Information für eine zelluläre Synthese von therapeutischen siRNAs enthält [19]. Derartige therapeutische DNA kann relativ leicht über virale

Pathologe 2008 • [Suppl 2] 29:187-190 DOI 10.1007/s00292-008-1030-2

(c) Springer Medizin Verlag 2008

H. Lage $\cdot$ P. Surowiak $\cdot$ P.S. Holm

YB-1 als potenzielles Ziel für die Tumortherapie

\section{Zusammenfassung}

In unterschiedlichen Tumorarten wird das pleiotrope 42-kDa-Protein YB-1 (Y-Box Protein 1) exprimiert. Es kann zytoplasmatisch lokalisiert sein oder sich im Zellkern befinden. Eine nukleäre Lokalisation wird insbesondere nach zellulärem Stress, wie z. B. Radiatio, Zytostatikabehandlung, Hyperthermie oder Virusinfektion, beobachtet. Im Zellkern kann YB-1 als Transkriptionsfaktor wirken und die Expression wichtiger tumorassoziierter Gene regulieren. So wurde z. B. im Mammakarzinom eine Korrelation zwischen YB-1-Expression und Her-2 und ERa (Östrogenrezeptor a) nachgewiesen. Nukleäres YB-1 erscheint daher als mögliches Target zur Inhibition Her-2 und ERa vermittelter Proliferationsignale; insbesondere bei Resistenz gegenüber gegen Her-2 gerichtete Therapeutika wie Trastuzumab. Darüber hinaus könnte YB-1 in eini- gen Tumoren an der Regulation des MDR1/ P-Glykoproteins (MDR1/P-Gp) beteiligt sein, welches mit dem klassischen Multidrug-Resistenz- (MDR-)Phänotyp assoziiert ist. Nukleäres YB-1 induziert zudem die Replikation von häufig in der Gentherapie eingesetzten Adenoviren Typ 5, d. h. YB-1 kann einen onkolytischen Effekt in mit Adenovirus behandelten YB-1 nukleär-positiven Tumorzellen bewirken. Neben seiner Bedeutung als prognostischer Faktor könnte die Bestimmung des zellulären YB-1-Status daher zukünftig die Voraussetzung für einen Einsatz einer Virotherapie oder einer viralen Gentherapie darstellen.

\section{Schlüsselwörter}

YB-1 - Zielgerichtete Therapie · Gentherapie · Virotherapie · Adenovirus

\section{YB-1 as a potential target in cancer therapy}

\section{Abstract}

The 42-kDa multifunctional cellular protein $\mathrm{Y}$ box protein 1 (YB-1) is expressed in various cancers. It is localized in the cytoplasm as well as in the nucleus. In particular, YB-1 is localized in the nuclear compartment following cellular stress, such as radiation, drug treatment, hyperthermia, or viral infection. Within the nucleus, YB- 1 can act as a transcription factor, and it is involved in the regulation of important cancer-associated genes. For example, YB-1 triggers the expression of Her-2 and estrogen receptor a (ERa) in breast cancer. Thus, nuclear YB-1 appears to be a potential target for the inhibition of Her-2- and ERa-dependent proliferation signals, particularly with regard to resistance to Her-2-targeting drugs such as trastuzumab. In some cancers, YB-1 may be involved in regulating MDR1/P-glycoprotein, mediating classical multidrug resistance (MDR). Furthermore, YB1 is involved in the replication of adenovirus type 5 , a commonly used vector in gene therapy. Thus, YB-1 can trigger an „oncolytic" effect in YB-1 nuclear positive cancer cells treated with adenoviruses. Besides its impact as a prognostic factor, in the future the diagnostics of cellular YB-1 status may provide the basis for a virotherapy or a gene therapy incorporating adenoviruses.

\section{Keywords}

YB-1 · Targeted therapy · Gene therapy · Virotherapy · Adenovirus 
Vektoren, z. B. Adenoviren, in Tumorzellen eingeschleust werden. Da YB-1 die Replikationsrate des RNAi vermittelnden Virus dramatisch erhöht, wird auch gleichzeitig die zelluläre Synthese der therapeutischen siRNAs drastisch verstärkt. Dass diese verstärkte siRNA-Synthese auch tatsächlich eine wesentliche Verstärkung der therapeutisch gewünschten biologischen Effekte nach sich zieht, konnte mit einem bereits detailliert charakterisierten Adenovirus [15], das die Synthese therapeutischer siRNAs zur Inhibition des MDR1/ P-Gp-vermittelten MDR-Phänotyps bewirkt, eindrucksvoll belegt werden [17].

\section{Fazit}

Zusammenfassend können aus den hier diskutierten Aspekten folgende Schlüsse gezogen werden:

- nukleäres YB-1 erscheint als mögliches Target zur Inhibition Her-2- und ERavermittelter Proliferationssignale bzw. Apoptosehemmung, z. B. bei Resistenz gegenüber Trastuzumab (Herceptin); - nukleäres YB-1 erscheint als mögliches Target zur Überwindung von MDR1/ P-Gp-vermittelter Multidrug-Resistenz fragwürdig; - nukleäres YB-1 besitzt E1A-like-Aktivität und kann damit die Replikation replikationsdefizienter $\triangle \mathrm{E} 1 \mathrm{~A}$-Adenoviren Typ 5 bewirken (Onkolyse); - nukleäres YB-1 gewährleistet einen therapeutisch nutzbaren onkolytischen Viruseffekt in vivo; und - neben seiner Bedeutung als Prognosefaktor könnte die Bestimmung des zellulären YB-1-Status zukünftig die Voraussetzung für einen möglichen Einsatz einer viralen Therapie darstellen. Die Bestimmung des zellulären YB-1-Status durch einen Pathologen könnte also zukünftig die Voraussetzung für die Planung einer Virotherapie bzw. einer auf Adenoviren basierenden Gentherapie darstellen.

\section{Korrespondenzadresse}

\section{Prof. Dr. Dr. H. Lage}

Institut für Pathologie

Charité-Universitätsmedizin Berlin, Campus Mitte

Charitéplatz 1, 10117 Berlin

hermann.lage@charite.de

Danksagung. Eigene Arbeiten zur Bedeutung von YB-1 als Target in der Tumortherapie werden vom
BMBF (Verbundvorhaben: Präklinische Untersuchungen zum Potenzial einer Therapie mit YB-1-abhängigen onkolytischen Adenoviren zur Therapie von Gehirntumoren; Förderkennzeichen: 01GU0615) finanziell unterstützt.

Interessenkonflikt. Zwei Autoren (H.L., P.S.H.) sind Gesellschafter und wissenschaftliche Berater bei der Firma Xvir Therapeutics GmbH in München, die einen onkolytischen Adenovirus zur Therapie des Glioblastoms entwickelt hat.

\section{Literatur}

1. Bader AG, Kang S, Zhao L, Vogt PK (2005) Oncogenic $\mathrm{PI} 3 \mathrm{~K}$ deregulates transcription and translation. Nat Rev Cancer 5: 921-929

2. Bargou RC, Jürchott K, Wagener C et al. (1997) Nuclear localization and increased levels of transcription factor YB-1 in primary human breast cancers are associated with intrinsic MDR1 gene expression. Nat Med 3: 447-450

3. Bergmann S, Royer-Pokora B, Fietze E et al. (2005) YB-1 provokes breast cancer through the induction of chromosomal instability that emerges from mitotic failure and centrosome amplification. Cancer Res 65: 4078-4087

4. Bieler A, Mantwill K, Holzmüller R et al. (2008) Impact of radiation therapy on the oncolytic adenovirus dl520: implications on the treatment of glioblastoma. Radiother Oncol 86: 419-427

5. Didier DK, Schiffenbauer J, Woulfe SL et al. (1988) Characterization of the CDNA encoding a protein binding to the major histocompatibility complex class II Y box. Proc Natl Acad Sci USA 85: 73227326

6. Faury D, Nantel A, Dunn SE et al. (2007) Molecular profiling identifies prognostic subgroups of pediatric glioblastoma and shows increased YB-1 expression in tumors. J Clin Oncol 25: 1196-1208

7. Fujii T, Kawahara A, Basaki Y et al. (2008) Expression of HER2 and estrogen receptor alpha depends upon nuclear localization of Y-box binding protein-1 in human breast cancers. Cancer Res 68: 1504-1512

8. Fujita T, Ito K, Izumi H et al. (2005) Increased nuclear localization of transcription factor Y-box binding protein 1 accompanied by up-regulation of P-glycoprotein in breast cancer pretreated with paclitaxel. Clin Cancer Res 11: 8837-8844

9. Giménez-Bonafé $P$, Fedoruk MN, Whitmore TG et al. (2004) YB-1 is upregulated during prostate cancer tumor progression and increases P-glycoprotein activity. Prostate 59: 337-349

10. Holm PS, Bergmann S, Jürchott K et al. (2002) YB1 relocates to the nucleus in adenovirus-infected cells and facilitates viral replication by inducing E2 gene expression through the E2 late promoter. J Biol Chem 277: 10427-10434

11. Holm PS, Lage H, Bergmann S et al. (2004) Multidrug-resistant cancer cells facilitate E1-independent adenoviral replication: impact for cancer gene therapy. Cancer Res 64: 322-328

12. Janz M, Harbeck N, Dettmar P et al. (2002) Y-box factor YB-1 predicts drug resistance and patient outcome in breast cancer independent of clinically relevant tumor biologic factors HER2, uPA and PAI1. Int J Cancer 97: 278-282

13. Jürchott K, Bergmann S, Stein U et al. (2003) YB-1 as a cell cycle-regulated transcription factor facilitating cyclin A and cyclin B1 gene expression. J Biol Chem 278: 27988-27996
14. Kamura T, Yahata H, Amada S et al. (1999) Is nuclear expression of $\mathrm{Y}$ box-binding protein-1 a new prognostic factor in ovarian serous adenocarcinoma? Cancer 85: 2450-2454

15. Kaszubiak A, Holm PS, Lage H (2007) Overcoming the classical multidrug resistance phenotype by adenoviral delivery of anti-MDR1 short hairpin RNAs and ribozymes. Int J Oncol 31: 419-430

16. Kaszubiak A, Kupstat A, Müller U et al. (2007) Regulation of MDR1 gene expression in multidrugresistant cancer cells is independent from YB-1. Biochem Biophys Res Commun 357: 295-301

17. Kaszubiak A, Surowiak P, Mantwill K et al. (2008) YB-1-mediated E1A-like activity in adenovirus infected multidrug-resistant cancer cells considerably enhance delivery and biological efficacy of therapeutic short hairpin RNAs. Proc Am Ass Cancer Res 49: 1365

18. Kohno K, Izumi H, Uchiumi T et al. (2003) The pleiotropic functions of the Y-box-binding protein, YB1. Bioessays 25: 691-698

19. Lage $H$ (2005) Potential applications of RNA interference technology in the treatment of cancer. Fut Oncol 1: 103-113

20. Mantwill K, Köhler-Vargas N, Bernshausen A et al. (2006) Inhibition of the multidrug-resistant phenotype by targeting YB-1 with a conditionally oncolytic adenovirus: implications for combinatorial treatment regimen with chemotherapeutic agents. Cancer Res 66: 7195-7202

21. Oda Y, Sakamoto A, Shinohara N et al. (1998) Nuclear expression of YB-1 protein correlates with $\mathrm{P}$ glycoprotein expression in human osteosarcoma. Clin Cancer Res 4: 2273-2277

22. Oda Y, Ohishi Y, Saito T et al. (2003) Nuclear expression of Y-box-binding protein-1 correlates with Pglycoprotein and topoisomerase II alpha expression, and with poor prognosis in synovial sarcoma. J Pathol 199: 251-258

23. Oda Y, Ohishi Y, Basaki Y et al. (2007) Prognostic implications of the nuclear localization of Y-box-binding protein- 1 and CXCR4 expression in ovarian cancer: their correlation with activated Akt, LRP/ MVP and P-glycoprotein expression. Cancer Sci 98: 1020-1026

24. Ranjan M, Tafuri SR, Wolffe AP (1993) Masking mRNA from translation in somatic cells. Genes Dev 7: $1725-1736$

25. Saji H, Toi M, Saji S et al. (2003) Nuclear expression of YB-1 protein correlates with P-glycoprotein expression in human breast carcinoma. Cancer Lett 190: 191-197

26. Shibahara K, Sugio K, Osaki T et al. (2001) Nuclear expression of the $\mathrm{Y}$-box binding protein, YB-1, as a novel marker of disease progression in non-small cell lung cancer. Clin Cancer Res 7: 3151-3155

27. Shibao K, Takano H, Nakayama Y et al. (1999) Enhanced coexpression of YB-1 and DNA topoisomerase II alpha genes in human colorectal carcinomas. Int J Cancer 83: 732-737

28. Stein U, Jürchott K, Walther W et al. (2001) Hyperthermia-induced nuclear translocation of transcription factor YB-1 leads to enhanced expression of multidrug resistance-related $A B C$ transporters. J Biol Chem 276: 28562-28569

29. Wu J, Lee C, Yokom D et al. (2006) Disruption of the Y-box binding protein-1 results in suppression of the epidermal growth factor receptor and HER-2. Cancer Res 66: 4872-4879

30. Wu J, Startford AL, Astanehe A, Dunn SE (2007) YB1 is a transcrition/translation factor that orchestrates the oncogenome by hardwiring signal transduction to gene expression. Translational Oncogenomics 2: 49-65 